\title{
Multi-transitional observations of methanol in Comet Hale-Bopp (1995 01)
}

\author{
M. Ikeda ${ }^{1, \star}$, K. Kawaguchi ${ }^{1, \star \star}$, S. Takakuwa ${ }^{1, \star \star \star}$, A. Sakamoto ${ }^{1}$, K. Sunada ${ }^{1}$, and T. Fuse ${ }^{2}$ \\ 1 Nobeyama Radio Observatory, Nobeyama, Minamimaki, Minamisaku, Nagano 384-1305, Japan \\ 2 Subaru Telescope, National Astronomical Observatory of Japan 650 North A'ohoku Place, Hilo, Hawaii 96720, USA
}

Received 2 August 1999 / Accepted 26 April 2002

\begin{abstract}
We have observed 9 rotational lines of methanol in comet Hale-Bopp (1995 O1) using the 45-m radio telescope at Nobeyama Radio Observatory in the frequency range 36-104 GHz. The observed transitions have upper state rotational energy levels of $E_{\mathrm{u}}=7-159 \mathrm{~K}$. Assuming the Haser model for density distribution, we estimated the excitation temperature and the production rate to be $T_{\mathrm{ex}}=81 \pm 8 \mathrm{~K}$ and $Q\left(\mathrm{CH}_{3} \mathrm{OH}\right)=(1.2 \pm 0.1) \times 10^{29}$ molecule s ${ }^{-1}$, respectively. We also observed the $\mathrm{HCN} J=1-0$ line at $88 \mathrm{GHz}$ to derive the $\mathrm{HCN}$ production rate. We compared the production rate of $\mathrm{CH}_{3} \mathrm{OH}$ with that of $\mathrm{HCN}$ and that of other molecules whose production rates have been determined previously. The abundance ratio $Q\left(\mathrm{CH}_{3} \mathrm{OH}\right) / Q(\mathrm{X})$ is estimated to be in the range 3-121, where $\mathrm{X}$ denotes $\mathrm{HCN}, \mathrm{HNC}, \mathrm{H}_{2} \mathrm{CO}, \mathrm{CS}$ and $\mathrm{CH}_{3} \mathrm{CN}$. When we compare these abundance ratios with those in interstellar clouds: the bipolar flow L1157 B1/B2, the star-forming region SgrB2(M) and the dark cloud TMC-1, they are close to the values in L1157 B1/B2 or in SgrB2(M). We found that the abundance ratio $Q\left(\mathrm{CH}_{3} \mathrm{OH}\right) / Q\left(\mathrm{H}_{2} \mathrm{O}\right)$ was similar to solid methanol ratio relative to water ice in interstellar medium. These results support the hypothesis that cometary nuclei are formed from dust grains outside of the primordial solar nebula so that the grain composition of the nebula remains in cometary nuclei.
\end{abstract}

Key words. comets: general - comets: individual: C/1995 O1(Hale-Bopp) - ISM: abundances

\section{Introduction}

Methanol is one of the most abundant interstellar molecules and was first detected in space more than 30 years ago (Ball et al. 1970). Not only gaseous methanol but also solid methanol has been detected in space. Several absorption features of methanol were found in W33A and other sources (Baas et al. 1988; Grim et al. 1991; Allamandola et al. 1992; Skinner et al. 1992; Sandford et al. 1993) by infrared observations. The distributions of methanol have also been observed toward from hot core, Orion-KL (Minh et al. 1993) to dark cloud, TMC-1 (Takakuwa et al. 1998; Takakuwa et al. 2000).

Methanol is expected to be present in comets if the cometary nucleus contains unmodified interstellar material, as is generally believed (Yamamoto 1985; Greenberg \& Hage 1990). The first radio detection of methanol lines in comet was made in comet Austin(1990 V) by Bockelée-Morvan et al. (1990). Subsequently methanol was also detected in

Send offprint requests to: M. Ikeda, e-mail: miho.ikeda@nao. ac . jp

* Present address: National Astronomical Observatory, Osawa 221-1, Mitaka, Tokyo 181-8588, Japan.

$\star \star$ Present address: Faculty of Science, Okayama University, Tsushima-naka 3-1-1, Okayama 700-8530, Japan.

$\star \star \star$ Present address: Institute of Astronomy and Astrophysics, Academia Sinica, PO Box 1-87, Nankang, Taipei 115-29, Taiwan. several other comets: in Levy(1990 XX) (Bockelée-Morvan et al. 1990), P/Swift-Tuttle(1992) (Bockelée-Morvan et al. 1994b), Hyakutake(C/1996 B2) (Womack et al. 1997), and Lee(C/1999 H1) (Biver et al. 2000). In comet HaleBopp $(\mathrm{C} / 1995$ O1), several methanol lines have been detected at $97,145,157,242,252,304$, and $307 \mathrm{GHz}$ by Biver et al. (1999). They observed the comet in a range of heliocentric distance, $r_{\mathrm{h}}$, from $7 \mathrm{AU}$ pre-perihelion to $4 \mathrm{AU}$ postperihelion and obtained a kinetic temperature of $T=(103 \pm$ 7) $r_{\mathrm{h}}^{(-1.10 \pm 0.08)} \mathrm{K}$ from average of pre and post-perihelion data of $\mathrm{CH}_{3} \mathrm{OH}$ and $\mathrm{CO}$.

In the present study, we have observed the $36,44,84,95$, 96 and $104 \mathrm{GHz}$ lines of methanol using the Nobeyama 45-m radio telescope. This is the first detection of 36 and $44 \mathrm{GHz}$ methanol lines in comet. From the multi-transitional observations of methanol, we have determined the excitation temperature and the production rate of methanol, and compare the abundance ratio of methanol in Hale-Bopp with observational results in interstellar medium.

\section{Observations}

Observations were made from the 9th to the 15th of February 1997, using the 45-m radio telescope at Nobeyama Radio 
Table 1. Characteristics of the NRO 45-m telescope.

\begin{tabular}{lll}
\hline \hline Frequency $(\mathrm{GHz})$ & beam width $\left({ }^{\prime \prime}\right)$ & main beam efficiency \\
\hline 36. & 47 & 0.87 \\
44. & 39 & 0.81 \\
84. & 18 & 0.48 \\
95. & 16 & 0.44 \\
96. & 16 & 0.44 \\
104. & 15 & 0.42 \\
\hline
\end{tabular}

Observatory ${ }^{1}$. During the observation period, comet HaleBopp was approaching the perihelion, with a geocentric distance, $\Delta$, and a heliocentric distance, $r_{\mathrm{h}}$, in the ranges of 1.851.73 AU and 1.27-1.21 AU, respectively.

Three SIS receivers $(100 \mathrm{GHz}, 80 \mathrm{GHz}$ and $40 \mathrm{GHz}$ ) were used at the frontend. The spectra of $\mathrm{CH}_{3} \mathrm{OH}$ and $\mathrm{HCN} J=$ 1-0 line at $88 \mathrm{GHz}$, which was observed every day during the observation period in order to check the system, were simultaneously observed by using $100 \mathrm{GHz}$ and $80 \mathrm{GHz}$ receivers or $100 \mathrm{GHz}$ and $40 \mathrm{GHz}$ receivers, respectively. The system temperatures of the three receivers were $300-500 \mathrm{~K}$, 280-550 K and 120-220 K, respectively, during the observations. The backend consists of two sets of acousto-optical spectrometers (AOSs). One is a bank of eight high-resolution AOSs with a frequency resolution of $37 \mathrm{kHz}$ and a bandwidth of $40 \mathrm{MHz}$ each. The other is a bank of eight wide-band AOSs with a frequency resolution of $250 \mathrm{kHz}$ and a bandwidth of $250 \mathrm{MHz}$ each. Pointing was checked every hour by observing nearest $\mathrm{SiO}$ maser emission from R-Aql. The estimated pointing accuracy was $\sim 5^{\prime \prime} \mathrm{rms}$. The observations were made toward the cometary nucleus in position-switching mode, and the typical off position was $15^{\prime}$ west of the source position. The beam widths and main beam efficiencies are summarized in Table 1. The on-source integration time was $0.24-2.6$ hours. The ephemeris and the radial velocity of comet Hale-Bopp were derived every hours. These values were calculated from the six orbital elements of the comet by taking into account perturbations from nine planets.

\section{Results}

Nine rotational transitions arising from methanol were detected. The corresponding line parameters are listed in Table 2. The observed line profiles of this molecule are shown in Fig. 1. Most of the lines have both blue and redshifted components, reflecting the expansion of $\mathrm{CH}_{3} \mathrm{OH}$ from the comet nucleus. The redshifted component was generally stronger than the blueshifted component, indicating asymmetric expansion of methanol. This trend has been reported in other observations. According to Irvine et al. (1998), time variation in line shape of HCN and HNC was recognized in Hale-Bopp and asymmetric line shape of $\mathrm{HCN}$ whose redshifted component was

\footnotetext{
${ }^{1}$ Nobeyama Radio Observatory is a branch of the National Astronomical Observatory of Japan, an interuniversity research institute operated by the Ministry of Education, Culture, Sports, Science and Technology, of Japan.
}

stronger than blueshifted component at February 1997 was reported. They concluded that this result suggests asymmetric out-gassing from the nucleus. Since methanol and HCN are both thought to be parent molecule, asymmetric line shape of methanol may also indicate asymmetric out-gassing from the nucleus. We estimated the $\mathrm{CH}_{3} \mathrm{OH}$ expansion velocity to be $0.86 \pm 0.20 \mathrm{~km} \mathrm{~s}^{-1}$ from the difference between the blue and the redshifted components assuming Gaussian profiles for the two lines. This value is consistent, within the limits of error, with the value of $1.0 \mathrm{~km} \mathrm{~s}^{-1}$ at $r_{\mathrm{h}}=1.24 \mathrm{AU}$, determined from the expansion velocity $V_{\exp }=(1.12 \pm 0.014) r_{\mathrm{h}}^{(-0.41 \pm 0.01)}$ suggested by Biver et al. (1999). Observed antenna temperatures and integrated intensities are listed in Table 2. The antenna temperatures of double-peaked lines are peak temperatures of redshifted component and those of single-peaked lines are peak temperatures of the line. The fluxes, $S_{v}=2 k v^{2} T_{\mathrm{b}} \Omega / c^{2}$ ( $\Omega$ : solid angle of the 45-m telescope beam, $T_{\mathrm{b}}=T_{\mathrm{a}}^{*} / \eta_{\mathrm{B}}$ : brightness temperature, $\eta_{\mathrm{B}}$ : beam efficiency), which are converted from the antenna temperatures, $T_{\mathrm{a}}^{*}$, for each frequency observed with the 45-m telescope, are shown in the last column of Table 2.

In order to obtain the production rate of $\mathrm{CH}_{3} \mathrm{OH}$, we analyzed the observed data using the method described by Schloerb et al. (1986). In this method it is assumed that the molecular distribution is described by the Haser model. That is, we assumed that the $\mathrm{CH}_{3} \mathrm{OH}$ molecules flow spherically away from the nucleus at a constant expansion velocity.

Assuming a scale length of $R_{\mathrm{d}}$ for photodissociation and a constant expansion velocity $V_{\text {exp }}$, the number density of the parent molecule can be written as:

$n(r)=\frac{Q}{4 \pi r^{2} V_{\exp }} \exp \left(-\frac{r}{R_{\mathrm{d}}}\right)$

where $r$ is the distance from the nucleus, and $Q$ is the production rate of the parent molecule. For $\mathrm{CH}_{3} \mathrm{OH}$, we adopted the photodissociation rate, $k_{\mathrm{p}}=1.3 \times 10^{-5} \mathrm{~s}^{-1}$, at the heliocentric distance of $1 \mathrm{AU}$ for the quiet sun (Bockelée-Morvan et al. 1994a), and $R_{\mathrm{d}}\left(=V_{\exp } / k_{\mathrm{p}} r_{\mathrm{h}}^{2}\right)$ was calculated to be $\sim 101700 \mathrm{~km}$. Assuming that the temperature and the expansion velocity are constant on the line of sight, integration of $n(r)$ along the line of sight, $z$, gives the column density

$N(a)=\int_{-\infty}^{\infty} n(r) \mathrm{d} z$,

where $N$ is the column density, $a$ is the projected distance from the nucleus, and $z$ is measured along the line of sight. Consider a point on the line of sight that has a distance $a$ from the nucleus when $\phi$ is the angle between the point and the plane which passes through the nucleus, we obtain

$N(a)=\frac{Q}{4 \pi V_{\exp } a} \int_{0}^{\pi} \exp \left(-\frac{a}{R_{\mathrm{d}} \sin \phi}\right) \mathrm{d} \phi$.

Assuming that the optical depth is small, the relation between the brightness temperature of the line and the column density averaged over the antenna beam, $\langle N\rangle$, is given as follows:

$$
\begin{aligned}
T_{\mathrm{b}}= & {\left[J\left(T_{\mathrm{ex}}\right)-J\left(T_{\mathrm{bg}}\right)\right] \frac{8 \pi^{3}}{3 h} \frac{\mu^{2} S\langle N\rangle}{\mathrm{d} v Z} } \\
& \times\left[\exp \left(\frac{h v}{k T_{\mathrm{ex}}}\right)-1\right] \exp \left(-\frac{E_{\mathrm{u}}}{k T_{\mathrm{ex}}}\right),
\end{aligned}
$$




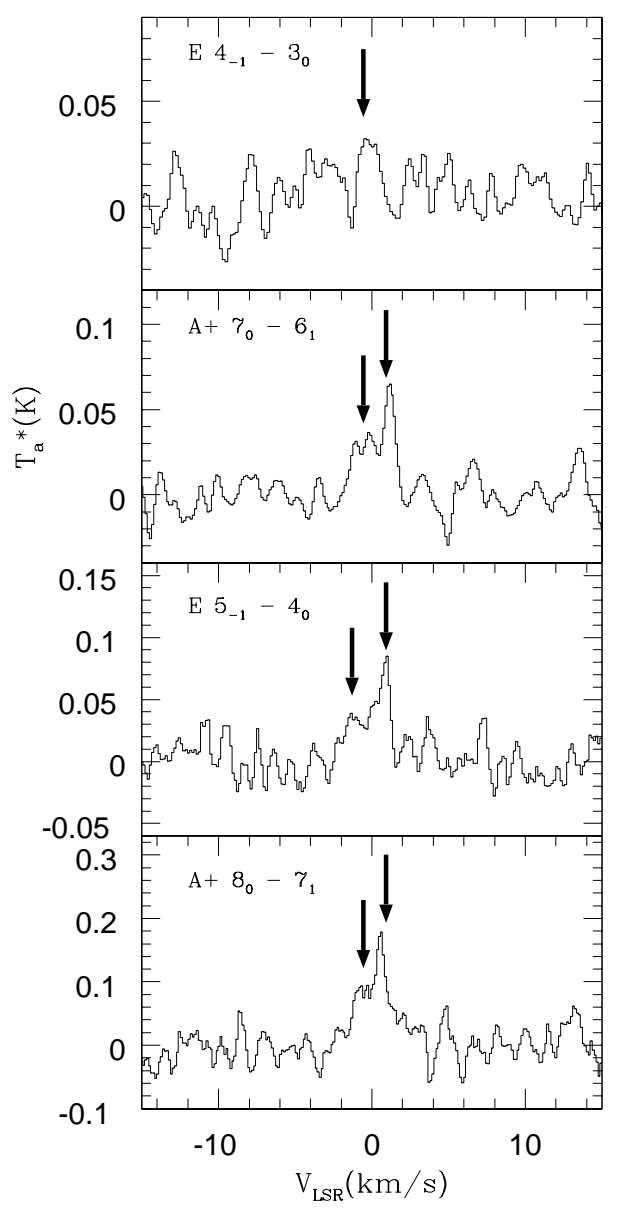

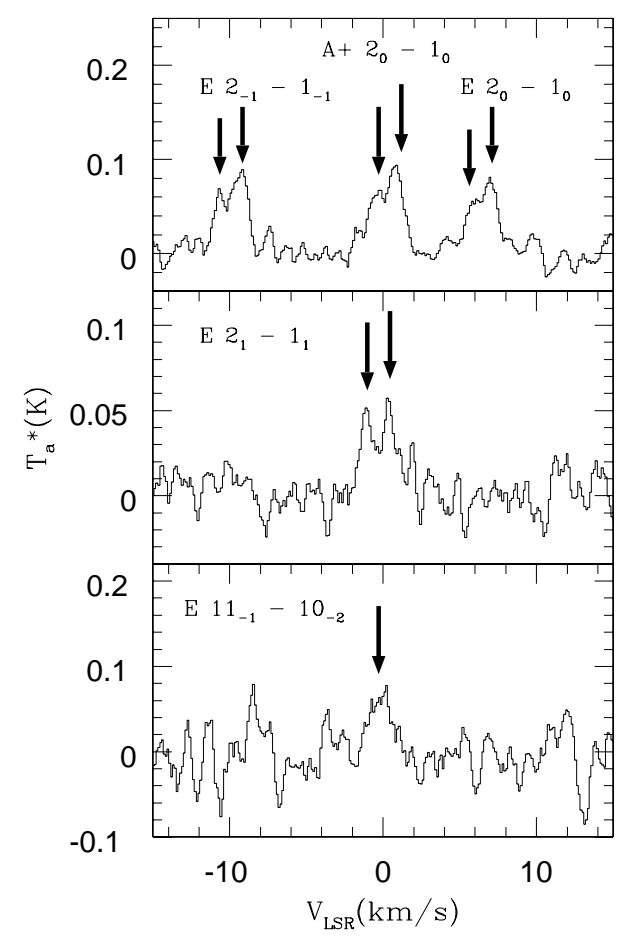

Fig. 1. Spectra of $\mathrm{CH}_{3} \mathrm{OH}$ toward Comet Hale-Bopp (1995 O1) (heliocentric distance is about 1.24 AU). The transition is shown at the upper left, center or right of the frame. Arrows indicate blue and redshifted components. where $J(T)=h v / k[\exp (h v / k T)-1]^{-1}, T_{\text {ex }}$ is the excitation temperature, $T_{\mathrm{bg}}$ is $2.7 \mathrm{~K}$ continuum background, $\mu$ is the permanent dipole moment, $S$ is the line strength, and $\mathrm{d} v$ is the line width. Since $\mathrm{CH}_{3} \mathrm{OH}$ is a molecule with internal rotation, the partition function, $Z$, is given as follows:

$Z=2\left[\frac{\pi}{A B C}\left(\frac{k T_{\mathrm{ex}}}{h}\right)^{3}\right]^{0.5}=1.2327 \times T_{\mathrm{ex}}^{1.5}$,

where $A, B$, and $C$ are the rotational constants of $\mathrm{CH}_{3} \mathrm{OH}$.

The antenna temperature produced by the brightness temperature distribution $T_{\mathrm{b}}(p)$ observed with a Gaussian beam of half-power beam width $\theta_{\mathrm{B}}$ and beam efficiency $\eta_{\mathrm{B}}$ is

$T_{\mathrm{a}}^{*}=\eta_{\mathrm{B}} \frac{8 \ln 2}{\theta_{\mathrm{B}}^{2}} \int_{0}^{\infty} T_{\mathrm{b}}(p) \exp \left[-4 \ln 2\left(\frac{p}{\theta_{\mathrm{B}}}\right)^{2}\right] p \mathrm{~d} p$

where $p(=a / \Delta: \Delta$ is geocentric distance $)$ is the angular size of $a$ and $T_{\mathrm{b}}(p)$ may be found by substituting $N(p)$ from Eq. (3) for $\langle N\rangle$ of Eq. (4). The integrated intensity of the line observed in the telescope's Gaussian beam is given as follows:

$$
\begin{aligned}
\int T_{\mathrm{a}}^{*} \mathrm{~d} v= & \eta_{\mathrm{B}} \frac{8 \ln 2}{\theta_{\mathrm{B}}^{2}}\left[J\left(T_{\mathrm{ex}}\right)-J\left(T_{\mathrm{bg}}\right)\right] \frac{8 \pi^{3}}{3 h} \frac{\mu^{2} S}{Z} \\
& \times \frac{Q}{4 \pi V_{\exp }}\left[\exp \left(\frac{h v}{k T_{\mathrm{ex}}}\right)-1\right] \exp \left(-\frac{E_{\mathrm{u}}}{k T_{\mathrm{ex}}}\right) \frac{F(p)}{\Delta}
\end{aligned}
$$

and $F(p)$ is

$$
F(p)=\int_{0}^{\infty} \mathrm{d} p \int_{0}^{\pi} \mathrm{d} \phi \exp \left[-\frac{p \Delta}{R_{\mathrm{d}} \sin \phi}\right] \exp \left[-4 \ln 2\left(\frac{p}{\theta_{\mathrm{B}}}\right)^{2}\right] .
$$

The excitation temperature, $T_{\mathrm{ex}}$, and the production rate, $Q$ were derived from the observed lines by adjusting these two parameters of Eq. (7) by a least-squares fit, where the integration of $F(p)$ is calculated numerically by Simpson's rule. We obtained these parameters to be $T_{\mathrm{ex}}=81 \pm 8 \mathrm{~K}$ and $Q\left(\mathrm{CH}_{3} \mathrm{OH}\right)=$ $(1.2 \pm 0.1) \times 10^{29}$ molecule s$^{-1}$. Biver et al. (1999) observed evolution increase of production rates of several molecules with heliocentric distance (See Fig. 3 of their paper). We investigated the heliocentric distance dependence of $Q\left(\mathrm{CH}_{3} \mathrm{OH}\right)$ from their data and calculated $Q\left(\mathrm{CH}_{3} \mathrm{OH}\right)$ at $r_{\mathrm{h}}=1.24 \mathrm{AU}$ to be $8.8 \times 10^{28}$ molecule $\mathrm{s}^{-1}$. This value is similar to our result. $T_{\mathrm{ex}}$ we obtained corresponds to the value of $81 \mathrm{~K}$ at $r_{\mathrm{h}}=1.24 \mathrm{AU}$, determined from the $T=(103 \pm 7) r_{\mathrm{h}}{ }^{(-1.10 \pm 0.08)}$ suggested by Biver et al. (1999).

We also observed the HCN $J=1-0$ line, with resolved hyperfine components, $F=1-1,2-1,0-1$ at $88.63 \mathrm{GHz}$. Each line has blue and redshifted velocity components with an average interval of $1.44 \mathrm{~km} \mathrm{~s}^{-1}$. We estimated the production rate of $\mathrm{HCN}$ from Feb. 10 data $\left(r_{\mathrm{h}}=1.26 \mathrm{AU}\right)$, of which the quality is high, using Eq. (7). Assuming an $\mathrm{HCN}$ photodissociation rate of $1.3 \times 10^{-5} \mathrm{~s}^{-1}\left(r_{\mathrm{h}}=1 \mathrm{AU}\right)$ for the quiet Sun and an excitation temperature equal to that for $\mathrm{CH}_{3} \mathrm{OH}$, the production rate of $\mathrm{HCN}$ is calculated to be 
Table 2. Observed $\mathrm{CH}_{3} \mathrm{OH}$ transitions with NRO 45-m telescope.

\begin{tabular}{lllllllll}
\hline \hline Transition & & $\begin{array}{l}\text { Rest freq. }^{\mathrm{a}} \\
{[\mathrm{GHz}]}\end{array}$ & $\begin{array}{l}E_{\mathrm{u}}^{\mathrm{b}} \\
{[\mathrm{K}]}\end{array}$ & $\begin{array}{l}\mu^{2} S^{\mathrm{c}} \\
{\left[\mathrm{D}^{2}\right]}\end{array}$ & $\begin{array}{l}\text { date } \\
(1997)\end{array}$ & $\begin{array}{l}T_{\mathrm{a}}^{*}(\mathrm{rms}) \\
{[\mathrm{mK}]}\end{array}$ & $\begin{array}{l}\int T_{\mathrm{a}}^{*} \mathrm{~d} v(\mathrm{rms}) \\
{\left[\mathrm{Km} \mathrm{s}^{-1}\right]}\end{array}$ & $\begin{array}{l}S_{v}(\mathrm{rms}) \\
{[\mathrm{mJy}]}\end{array}$ \\
\hline $4_{-1}-3_{0}$ & $\mathrm{E}$ & 36.169290 & 28.789 & 2.518 & Feb.11-13 & $32(11)$ & $0.049(0.008)$ & $87(30)$ \\
$7_{0}-6_{1}$ & $\mathrm{~A}^{+}$ & 44.069476 & 64.981 & 6.138 & Feb.9-10 & $68(16)$ & $0.107(0.013)$ & $203(48)$ \\
$5_{-1}-4_{0}$ & $\mathrm{E}$ & 84.521206 & 40.391 & 3.083 & Feb.15 & $87(22)$ & $0.137(0.019)$ & $343(87)$ \\
$8_{0}-7_{1}$ & $\mathrm{~A}^{+}$ & 95.169516 & 83.539 & 7.221 & Feb.13 & $179(28)$ & $0.303(0.028)$ & $771(121)$ \\
$2_{-1}-1_{-1}$ & $\mathrm{E}$ & 96.739363 & 12.542 & 1.213 & Feb.14 & $83(16)$ & $0.141(0.014)$ & $369(71)$ \\
$2_{0}-1_{0}$ & $\mathrm{~A}^{+}$ & 96.741377 & 6.965 & 1.617 & Feb.14 & $97(16)$ & $0.169(0.014)$ & $431(71)$ \\
$2_{0}-1_{0}$ & $\mathrm{E}$ & 96.744549 & 20.090 & 1.617 & Feb.14 & $88(16)$ & $0.173(0.014)$ & $391(71)$ \\
$2_{1}-1_{1}$ & $\mathrm{E}$ & 96.755507 & 28.011 & 1.244 & Feb.14 & $59(16)$ & $0.112(0.016)$ & $263(71)$ \\
$11_{-1}-10_{-2}$ & $\mathrm{E}$ & 104.300396 & 158.637 & 3.414 & Feb.15 & $84(28)$ & $0.113(0.022)$ & $400(133)$ \\
\hline
\end{tabular}

a Frequencies from Xu \& Lovas (1997).

b Energy of the upper level of the transition.

c Square of dipole moment times line strength.

$Q(\mathrm{HCN})=7.6 \times 10^{27}$ molecule $\mathrm{s}^{-1}$ from the integrated intensity in which the three hyperfine components were added. The integrated intensity, $\int T_{\mathrm{a}}^{*} \mathrm{~d} v$, and $\mu^{2} S$ which comprised the three hyperfine components are $1.645 \mathrm{~K} \mathrm{~km} \mathrm{~s}^{-1}$ and $8.9 \mathrm{D}^{2}$, respectively. The upper energy of each hyperfine component and the beam efficiency are $4.3 \mathrm{~K}$ and 0.48 , respectively. The obtained production rate is lower than the value of $(1.7 \pm 0.3) \times 10^{28}$ molecule $\mathrm{s}^{-1}$ at $r_{\mathrm{h}}=0.98 \mathrm{AU}$ (Hirota et al. 1999), probably because of the larger heliocentric distance in the present observation. When we investigate the heliocentric distance dependence of $Q(\mathrm{HCN})$ from the data of Biver et al. (1999), $Q(\mathrm{HCN})$ at

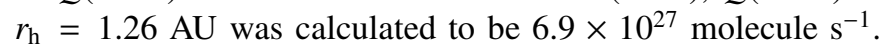
This value is similar to our result.

\section{Discussion and summary}

The sublimation temperatures of $\mathrm{HCN}$ and $\mathrm{CH}_{3} \mathrm{OH}$ are estimated to be $95 \mathrm{~K}$ and $99 \mathrm{~K}$ (Yamamoto 1985), respectively at a gas density of $10^{13} \mathrm{~cm}^{-3}$. Both molecules are thought to be parent species and to be sublimated similarly. Therefore, the production rates of $\mathrm{HCN}$ and $\mathrm{CH}_{3} \mathrm{OH}$ may reflect the original abundances in the comet nucleus. The abundance ratio $\left[\mathrm{CH}_{3} \mathrm{OH}\right] /[\mathrm{HCN}]$ was obtained from $Q\left(\mathrm{CH}_{3} \mathrm{OH}\right)$ at $r_{\mathrm{h}}=$ $1.24 \mathrm{AU}$ and $Q(\mathrm{HCN})$ at $r_{\mathrm{h}}=1.26 \mathrm{AU}$ to be 16 . This value agrees within a factor of 2 with the value of 9.6 at $r_{\mathrm{h}} \sim$ 1 AU obtained by Bockelée-Morvan et al. (2000). When we compare our abundance ratios with those of previous comets, $\left[\mathrm{CH}_{3} \mathrm{OH}\right] /[\mathrm{HCN}]=28$ in Levy (Crovisier et al. 1993), 80 in Austin (Crovisier et al. 1993), and 40-70 in Swift-Tuttle (Despois et al. 1996) are larger than our result, but 20 in Hyakutake (Lis et al. 1997) is similar to our result.

Solid methanol has been identified toward W33A and several other protostellar objects (Baas et al. 1988; Grim et al. 1991; Allamandola et al. 1992; Skinner et al. 1992; Sandford et al. 1993), with an abundance of 7 to $40 \%$ relative to water ice. Dello Russo et al. (2000) obtained water production rate to be $Q\left(\mathrm{H}_{2} \mathrm{O}\right)=8.35 \times 10^{30} r_{\mathrm{h}}^{-1.88}$. We obtained $Q\left(\mathrm{H}_{2} \mathrm{O}\right)$ at $r_{\mathrm{h}}=1.24 \mathrm{AU}$ to be $5.6 \times 10^{30}$ molecule $\mathrm{s}^{-1}$ from this equation. Therefore, $\left[\mathrm{CH}_{3} \mathrm{OH}\right] /\left[\mathrm{H}_{2} \mathrm{O}\right]$ at $r_{\mathrm{h}}=1.24 \mathrm{AU}$ is $2 \%$. This value agrees to within a factor of 4 with the smallest value in interstellar ice.

We compared the abundance ratios in Hale-Bopp with those in interstellar clouds. We obtained $\mathrm{CH}_{3} \mathrm{OH}$ abundance ratio relative to other molecules, $\left[\mathrm{CH}_{3} \mathrm{OH}\right] /[\mathrm{X}]$, where $\mathrm{X}$ indicates $\mathrm{HCN}, \mathrm{HNC}, \mathrm{H}_{2} \mathrm{CO}, \mathrm{CS}$ and $\mathrm{CH}_{3} \mathrm{CN}$. There are no observational molecular data at $r_{\mathrm{h}} \sim 1.24 \mathrm{AU}$ except for $\mathrm{CH}_{3} \mathrm{OH}$ and $\mathrm{HCN}$, so that we investigated the heliocentric distance dependence of $Q(\mathrm{X})$ from the data of Biver et al. (1999) and calculated $Q(\mathrm{X})$ at $r_{\mathrm{h}}=1.24 \mathrm{AU}$. The sublimation temperatures, production rates, and abundance ratios of molecules in HaleBopp are listed in Table 3 with the abundances in interstellar clouds for comparison. The abundance ratios in Hale-Bopp were obtained as ratios between the production rates and those in interstellar clouds were obtained as ratios between the column densities. The ratios $\left[\mathrm{CH}_{3} \mathrm{OH}\right] /[\mathrm{X}]$ in Hale-Bopp agree within a factor of 1-4 with those in the bipolar flow L1157 $\mathrm{B} 1 / \mathrm{B} 2$ or the star-forming region $\mathrm{SgrB} 2(\mathrm{M})$. On the other hand, the ratios $\left[\mathrm{CH}_{3} \mathrm{OH}\right] /[\mathrm{X}]$ in the dark cloud TMC-1 are $2-3$ orders of magnitude smaller than those in Hale-Bopp.

In the bipolar flow L1157 B1/B2, methanol which is thought to be evaporated from dust grain is enhanced (Bachiller et al. 1997). In the star-forming region SgrB2, many organic molecules are enhanced and thought to be evaporated from dust grain (Miao et al. 1995). Methanol is thought to be one of such molecules. On the other hand, the kinetic temperature in dark cloud such as TMC-1 is low, molecules cannot be evaporated from dust grain. Therefore, methanol abundance ratios relative to other molecules in Hale-Bopp are similar to values in the molecular cloud in which methanol can evaporated from dust grain. This fact supports the hypothesis that when cometary nuclei are formed from dust grains outside of a primordial solar nebula, the nascent grain composition of the solar nebula may remain in the cometary nuclei.

Acknowledgements. We would like to thank all the staff at the Nobeyama Radio Observatory for their assistance with our observations. We also thank G. A. Blake of the California Institute of Technology, N. Ukita of the Nobeyama Astronomical Observatory of Japan, T. J. Millar of University of Manchester Institute of Science and 
Table 3. Sublimation temperatures, production rates $(Q)$ of molecules in Hale-Bopp, and abundance ratios $\left[\mathrm{CH}_{3} \mathrm{OH}\right] /[\mathrm{X}]$ in $\mathrm{Hale-Bopp}$ and interstellar clouds.

\begin{tabular}{|c|c|c|c|c|c|c|}
\hline \multirow[b]{2}{*}{ Molecule $^{\mathrm{a}}$} & \multirow[b]{2}{*}{$\begin{array}{l}\text { Subli. Temp. } \\
{[\mathrm{K}]}\end{array}$} & \multicolumn{2}{|c|}{ Comet Hale-Bopp } & \multicolumn{2}{|c|}{ interstellar space } & \multirow[b]{2}{*}{$\begin{array}{l}{\left[\mathrm{CH}_{3} \mathrm{OH}\right] /[\mathrm{X}]} \\
\text { TMC- } 1^{\mathrm{e}}\end{array}$} \\
\hline & & $\begin{array}{l}Q(1.24 \mathrm{AU}) \\
{\left[\mathrm{mol} \mathrm{s}^{-1}\right]}\end{array}$ & {$\left[\mathrm{CH}_{3} \mathrm{OH}\right] /[\mathrm{X}]$} & $\begin{array}{l}{\left[\mathrm{CH}_{3} \mathrm{OH}\right] /[\mathrm{X}]} \\
\mathrm{L} 1157 \mathrm{~B} 1 / \mathrm{B} 2^{\mathrm{c}}\end{array}$ & $\begin{array}{l}{\left[\mathrm{CH}_{3} \mathrm{OH}\right] /[\mathrm{X}]} \\
\operatorname{SgrB} 2(\mathrm{M})^{\mathrm{d}}\end{array}$ & \\
\hline $\mathrm{CH}_{3} \mathrm{OH}$ & 99 & $1.2 \times 10^{29}$ & - & - & - & - \\
\hline $\mathrm{HCN}$ & 95 & $7.6 \times 10^{27 f}$ & 16 & $3 / 41$ & $1-6$ & $0.11^{\mathrm{h}}$ \\
\hline $\mathrm{HNC}$ & - & $1.1 \times 10^{27}$ & 109 & $36 / 480$ & $3-19$ & $0.07^{\mathrm{h}}$ \\
\hline$\left(\mathrm{H}_{2} \mathrm{CO}\right)$ & 64 & $3.8 \times 10^{28}$ & 3 & $3 / 60$ & $2-13$ & 0.05 \\
\hline (CS) & - & $5.6 \times 10^{27}$ & 21 & $6 / 120$ & $1-4$ & 0.4 \\
\hline $\mathrm{CH}_{3} \mathrm{CN}$ & 91 & $9.9 \times 10^{26}$ & 121 & - & $70-470^{\mathrm{g}}$ & 4 \\
\hline
\end{tabular}

a Molecules in parentheses are thought to be "daughter" molecules.

b Yamamoto (1985).

c Observed molecular abundances in the bipolar flow by Bachiller et al. (1997).

d Observed molecular abundances in the star-forming region by Sutton et al. (1991).

e Observed molecular abundances in the dark cloud by Ohishi et al. (1998).

f Obtained from data at $r_{\mathrm{h}}=1.26 \mathrm{AU}$.

g $N\left(\mathrm{CH}_{3} \mathrm{CN}\right)$ was adopted from Turner (1991).

h $N(\mathrm{HCN})$ and $N(\mathrm{HNC})$ were estimated from $\mathrm{H}^{13} \mathrm{CN}$ and $\mathrm{HN}^{13} \mathrm{C}$ data of Hirota et al. $(1998)$ assuming ${ }^{12} \mathrm{C} /{ }^{13} \mathrm{C}=60$.

Technology, and M. Ohishi of the National Astronomical Observatory of Japan for helpful discussion. This study is partly supported by a Grant-in-Aid from the Ministry of Education, Science, Sports and Culture of Japan(No 09044108).

\section{References}

Allamandola, L. J., Sandford, S. A., Tielens, A. G. G. M., \& Herbst, T. M. 1992, ApJ, 399, 134

Baas, F., Grim, R. J. A., Schutte, W. A., et al. 1988, in Proceeding of the Conference, Manchester, England (Cambridge and New York, Cambridge University Press), 55

Bachiller, R., \& Pérez Gutiérrez, M. 1997, ApJ, 487, L93

Ball, J. A., Gottlieb, C. A., Lilley, A. E., \& Radford, H. E. 1970, ApJ, $162, \mathrm{~L} 203$

Biver, N., Bockelée-Morvan, D., Colom, P., et al. 1999, Earth, Moon and Planets, 78, 5

Biver, N., Bockelée-Morvan, D., Crovisier, J., et al. 2000, AJ, 120, 1554

Bockelée-Morvan, D., Crovisier, J., Colom, P., et al. 1990, in ESA, Formation of Stars and Planets, and the Evolution of the Solar System, 143

Bockelée-Morvan, D., Crovisier, J., Colom, P., \& Despois, D. 1994a, A\&A, 287, 647

Bockelée-Morvan, D., Padman, R., Davies, J. K., \& Crovisier, J. 1994b, Planet. Space Sci., 42, 655

Bockelée-Morvan, D., Lis, D. C., Wink, J. E., et al. 2000, A\&A, 353, 1101

Crovisier, J., Bockelée-Morvan, D., Colom, P., et al. 1993, A\&A, 269, 527

Despois, D., Biver, N., Bockelée-Morvan, D., et al. 1996, Planet. Space Sci., 44, 529

Dello Russo, N., Mumma, M. J., DiSanti, M. A., et al. 2000, Icarus, 143,324
Grim, R. J. A., Baas, F., Geballe, T. R., et al. 1991, A\&A, 243, 473

Greenberg, J. M., \& Hage, J. I. 1990, ApJ, 361, 260

Hirota, T., Yamamoto, S., Mikami, H., \& Ohishi, M. 1998, ApJ, 503, 717

Hirota, T., Yamamoto, S., Kawaguchi, K., et al. 1999, ApJ, 520, 895

Irvine, W. M., Dickens, J. E., Lovell, A. J., et al. 1998, Chemistry and Physics of Molecules and Grains in Space, Faraday Discussions No. 109, The Faraday Division of the Royal Society of Chemistry, London, 475

Lis, D. C., Keene, J., Young, K., et al. 1997, Icarus, 130, 355

Miao, Y., Mehringer, D. M., Kuan, Y.-J., \& Snyder, L. E. 1995, ApJ, 445, L59

Minh, Y. C., Ohishi, M., Roh, D. G., et al. 1993, ApJ, 411, 773

Ohishi, M., \& Kaifu, N. 1998, Chemistry and Physics of Molecules and Grains in Space, Faraday Discussions No. 109, The Faraday Division of the Royal Society of Chemistry, London, 205

Sandford, S. A., Allamandola, L. J., \& Geballe, T. R. 1993, Science, 262, 400

Schloerb, F. P., Kinzel, W. M., Swade, D. A., \& Irvine, W. M. 1986, ApJ, 310, L55

Skinner, C. J., Tielens, A. G. G. M., Barlow, M. J., \& Justtanont, K. 1992, ApJ, 399, L79

Sutton, E. C., Jaminet, P. A., Danchi, W. C., \& Blake, G. A. 1991, ApJS, 77, 255

Takakuwa, S., Mikami, H., \& Saito, M. 1998, ApJ, 501, 723

Takakuwa, S., Mikami, H., Saito, M., \& Hirano, N. 2000, ApJ, 542, 367

Turner, B. E. 1991, ApJS, 76, 617

Womack, M., Stern, S. A., \& Festou, M. C. 1997, Planet. Space Sci., 45,711

Xu, L.-H., \& Lovas, F. J. 1997, J. Phys. Chem. Ref. Data, 26, 17

Yamamoto, T. 1985, A\&A, 142, 31 